\title{
Influência do Número de Séries e Tempo de Alongamento Estático Sobre a Flexibilidade dos Músculos Isquiotibiais em Mulheres Sedentárias
}

\author{
Influence of the Number of Sets and Time of Static Stretching on the \\ Flexibility of Hamstring Muscles in Sedentary Women
}

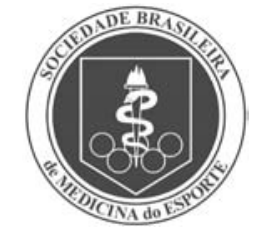

Artigo Original
Mariana Vita Milazzotto'

Luciana Gruba Corazzina'

Richard Eloin Liebano

1. Universidade Cidade de São Paulo (Unicid), São Paulo, SP - Brasil. 2. Universidade Cidade de São Paulo (Unicid), São Paulo, SP - Brasil e Universidade Federal de São Paulo - Escola Paulista de Medicina (Unifesp-EPM), São Paulo, SP - Brasil.

Endereço para correspondência: Richard Eloin Liebano -

Universidade Cidade de São Paulo - Departamento de Fisioterapia. Rua Cesário Galeno, 448/475 03071-000 - São Paulo, SP - Brasil. Tel.: (11) 2178-1479.

E-mail: liebano@gmail.com

Submetido em: 28/09/2008 Versão final recebida em: 09/06/2009 Aceito em: 15/06/2009

\begin{abstract}
RESUMO
Introdução e objetivo: Os exercícios de alongamento são frequentemente utilizados em programas de reabilitação e na área desportiva, porém, a duração ideal e o número de séries ainda não foram determinados. O objetivo do estudo foi avaliar os efeitos de 10 séries de 30 segundos e três séries de três minutos de alongamento estático passivo na flexibilidade dos músculos isquiotibiais, comparando diferentes volumes de alongamento dentro de 10 minutos. Além disso, verificar se existe diferença entre os dois programas de alongamento ao término do protocolo e após cinco meses. Métodos: 25 mulheres (17 a 25 anos) foram distribuídas aleatoriamente em três grupos: grupo GC (controle), G30 e G3. O G30 alongou 10 X 30 segundos e o G3 3 X 3 minutos. O alongamento foi realizado por meio de um sistema de polias, utilizando-se 10\% do peso corporal da voluntária. Este protocolo durou 6 semanas. As mensurações foram feitas no início do protocolo (Av1), após seis semanas (Av2) e após cinco meses (Av3). Foi mensurada a amplitude de movimento (ADM) de extensão do joelho com $90^{\circ}$ de flexão do quadril. A análise de variância (Anova) com dois fatores cruzados de medidas repetidas foi usada com nível de significância de 5\% ( $p \leq 0,05)$. Resultados: Não houve diferença estatisticamente significante na ADM de joelho entre 30 segundos (G30) e três minutos (G3) após seis semanas (Av2) ( $p>0,05)$ e após cinco meses (Av3) ( $p>0,05)$. Tanto o G30 quanto o G3 apresentaram aumento da flexibilidade ao término do protocolo (Av1xAv2) $(p<0,05)$ e após cinco meses (Av1xAv3) ( $p<$ 0,05). Conclusões: Dez séries de 30 segundos e três séries de três minutos podem aumentar a flexibilidade dos isquiotibiais e manter a flexibilidade adquirida após cinco meses do término da intervenção.
\end{abstract}

Palavras-chave: técnicas de fisioterapia, alongamento passivo, amplitude de movimento articular, treinamento.

\begin{abstract}
Introduction and objective: Stretching exercises are largely used in rehabilitation programs, but the ideal duration and the number of sets have not been determined yet. The purpose of this study was to evaluate the effects of 10 sets-30 seconds and 3 sets-3 minutes of static stretch on flexibility of hamstring muscles, comparing different volumes within 10 minutes. Moreover, to verify the difference between the two stretching programs. Method: 25 women ( 17 to 25 years old) were randomly assigned to one of 3 groups: group GC (control), G30 and G3. G30 stretched $10 \times 30$ seconds and G3 $3 \times 3$ minutes. In all volunteers the stretching was performed by a pulley system, using weight corresponding to $10 \%$ of their body weight. This protocol took 6 weeks. Measurements were taken at the beginning of the protocol (Av1), after 6 weeks (Av2), and after 5 months (Av3). Flexibility of the hamstring was determined by measuring knee extension range of movement (ROM) with $90^{\circ}$ of hip flexion. A two-way repeated measures Analysis of Variance (Anova) was used and significance level was set at $5 \%(p \leq 0.05)$. Results: There was no statistically significant difference between 30 seconds (G30) and 3 minutes (G3) in knee extension ROM after 6 weeks (Av2) ( $p>0.05$ ) and neither after 5 months (Av3) ( $p>0.05)$. Both the $G 30$ as the $G 3$ showed increasing flexibility at the end of protocol (Av1xAv2) $(p<0.05)$ and after 5 months (Av1xAv3) $(p<0.05)$. Conclusion: Stretching sets of 30 seconds and 3 minutes can increase the hamstrings flexibility and keep the flexibility obtained after 5 months from the end of intervention. There was no difference on the flexibility obtained with sets of 30 seconds or 3 minutes.
\end{abstract}

Keywords: physical therapy techniques; passive stretching; range of motion; training.

\section{INTRODUÇÃO}

A flexibilidade é dependente da viscoelasticidade do tecido conjuntivo(1). Ela é componente da função humana normal e, quando limitada, predispõe o indivíduo a diversas lesões musculoesqueléticas. Por isso, é de fundamental importância para os profissionais da área de fisioterapia e do esporte a avaliação da flexibilidade dos músculos por meio da mensuração da amplitude de movimento (ADM) ${ }^{(2,3)}$. A reali- zação dos exercícios de alongamento, para aumento da flexibilidade muscular, é importante para reduzir as lesões e aumentar a performance nos esportes ${ }^{(4)}$. Para tanto, precisa-se escolher um programa de alongamento adequado, com frequência e número de repetições definidos, além do tempo adequado de sustentação do alongamento ${ }^{(1,3,5,6)}$.

O alongamento estático passivo é o método de mais fácil realização e o mais frequentemente utilizado ${ }^{(1,2,7-9)}$. Decoster et al. ${ }^{(10)}$ afirmam, em sua 
revisão de literatura, que os indivíduos que realizam o alongamento estático alcançam ganhos maiores de amplitude de movimento quando comparados com os que empregam as técnicas de facilitação neuromuscular proprioceptiva. No entanto, para os atletas obterem as vantagens dos ganhos de flexibilidade com o alongamento estático, é importante saber por quanto tempo duram os efeitos ${ }^{(11)}$. Estudos que avaliam a plasticidade e quanto tempo esta perdura, após um treino de alongamento, não foram encontrados. Somente Magnusson et al. ${ }^{(12)}$ descrevem, de maneira geral, que o indivíduo sedentário deveria alongar-se pelo menos uma vez ao dia, três ou cinco dias por semana, e manter o alongamento por maior tempo possível a fim de obter ganho ou manter a flexibilidade.

Borms et al. ${ }^{(13)}$ compararam os efeitos de séries de 10, 20 e 30 segundos em alongamento estático dos isquiotibiais e nenhuma diferença significante foi encontrada entre os três grupos. Alguns outros autores também compararam séries de alongamento com diferentes tempos de duração ${ }^{(7,8)}$, porém, não foram encontrados trabalhos que utilizassem tempos de alongamento estático dos músculos isquiotibiais acima de dois minutos. Além disso, nos estudos encontrados, a flexibilidade é apenas avaliada logo após o programa de alongamento, não sendo possível, dessa maneira, determinar a duração dos resultados obtidos. Dessa forma, o objetivo deste estudo foi avaliar os efeitos de 10 séries de 30 segundos e três séries de três minutos de alongamento estático passivo na flexibilidade dos músculos isquiotibiais, comparando diferentes volumes de alongamento dentro de 10 minutos. Além disso, verificar se existe diferença entre os dois programas de alongamento ao término do protocolo e após cinco meses.

\section{MÉTODOS}

Os protocolos foram aplicados em 30 voluntárias universitárias do curso de Fisioterapia da Universidade Cidade de São Paulo, sedentárias, com média de idade de $21 \pm 4$ anos, sendo que apenas 25 concluíram o estudo. No início do trabalho foi explicado a todas as voluntárias que só participariam da pesquisa as que não realizassem qualquer tipo de atividade física durante o período do estudo. Além disso, o termo de consentimento livre e esclarecido continha de forma clara essa necessidade. Em todas as avaliações as voluntárias foram questionadas quanto à pratica de atividades físicas para garantir que não realizassem atividades físicas durante o período do estudo. Se caso a resposta fosse afirmativa quanto à prática da atividade, a voluntária era automaticamente excluída do estudo. O trabalho foi aceito pelo comitê de Ética e Pesquisa da Universidade Cidade de São Paulo.

Participaram do estudo as voluntárias que apresentaram encurtamento bilateral da musculatura posterior da coxa; considerou-se encurtamento quando a flexão ativa de quadril, com o joelho estendido, era menor que $90^{\circ(14)}$. Não foram incluídas as que apresentavam gravidez, doenças vasculares, desordens neuromusculares, lesões em quadril, joelho e/ou tornozelo, tanto articulares quanto musculares, e doença maligna. Foram excluídas as que tiveram mais de três faltas durante o período do estudo.

As voluntárias foram distribuídas de forma aleatória em três grupos: grupo controle (GC), formado por nove indivíduos, que não foram submetidos a nenhum tipo de intervenção; G30, formado por sete indivíduos, que foram alongados por 30 segundos, realizando 10 repetições, com intervalos de 30 segundos entre elas, totalizando 10 minutos de alongamento; G3, formado por nove indivíduos, que foram alongados por três minutos, realizando três repetições, com intervalos de 30 segundos entre elas, totalizando 10 minutos de alongamento.

Um goniômetro universal da marca $\mathrm{Carci}^{\circledR}$ foi utilizado para avaliar a ADM da articulação do joelho ${ }^{(2,3,5,7-9)}$ de ambos os membros inferiores, pré-intervenção (Av1), pós (Av2) e após cinco meses do término da mesma (Av3). A ADM das voluntárias foi mensurada por um avaliador cego. Todas as avaliações foram realizadas pelo mesmo avaliador. Foram colhidas três medidas do ângulo articular pesquisado e foi utilizada a média entre as três medidas.
Para a execução dessas avaliações, as voluntárias foram posicionadas em decúbito dorsal, fixadas à maca com faixas de Velcro e com o membro inferior esquerdo (aleatoriamente escolhido) livre. Uma das faixas foi fixada na região da tuberosidade da tíbia direita e a outra, a aproximadamente $15 \mathrm{~cm}$ da espinha ilíaca anterossuperior direita. Solicitou-se à voluntária, a partir de $90^{\circ}$ de flexão do quadril, uma extensão ativa do joelho. Definiu-se como ponto de mensuração o ângulo articular presente quando a voluntária referisse estar sentindo leve desconforto sem dor, ou tensão na região posterior da coxa. A posição neutra foi considerada com o quadril e joelhos em flexão de $90^{\circ}$ e a extensão total de joelho, como $90^{\circ}$. O tornozelo permaneceu relaxado durante toda a avaliação(5,14).

No início de cada sessão realizou-se aquecimento na bicicleta ergométrica, sem carga, por cinco minutos. Para realização dos programas de alongamento, as voluntárias foram fixadas à maca em decúbito dorsal, exatamente na mesma posição de quando avaliadas, conforme ilustrado na figura 1. O alongamento estático passivo foi realizado por um sistema com uma polia e uma corda. Uma das extremidades da corda foi fixada ao tornozelo esquerdo da voluntária, enquanto a outra, a um peso equivalente a $10 \%$ da massa corpórea da mesma ${ }^{(5,15,16)}$. De maneira ativa a voluntária realizava a extensão total do joelho e flexionava o quadril até que sentisse tensão provocada pelo alongamento, conforme ilustrado na figura 1. Em seguida, as voluntárias eram orientadas a relaxar e permitir que o peso, fixo à corda que passava pelo sistema das polias, levasse o membro inferior para a posição do alongamento e mantivessem essa posição durante o tempo proposto pelo protocolo.

O treino de flexibilidade foi realizado três vezes por semana, durante seis semanas.

\section{ANÁLISE ESTATÍSTICA}

A análise estatística foi realizada utilizando o programa SPSS (Statistical Package for Social Sciences) for Windows versão 14.0. Inicialmente, foi realizada a análise descritiva dos dados e o teste de normalidade de Kolmogorov-Smirnov.

A comparação entre as cargas utilizadas para o alongamento nos dois grupos (G30 e G3) foi realizada por meio do teste $t$ para dados independentes.

Os ângulos articulares foram comparados entre os grupos e entre as avaliações através de uma Anova com dois fatores cruzados de medidas repetidas. Consideraram-se os grupos como o fator entre indivíduos e as avaliações como o fator intraindivíduos. E, em caso de interação significativa, a diferença entre os grupos (em cada avaliação) e a diferença entre as avaliações (dentro de cada grupo) foram identificadas através das médias estimadas e dos intervalos de confiança corrigidos pelo método LSD (least significant difference).

Para todas as comparações foi utilizado índice de significância de $p \leq 0,05(5 \%)$.

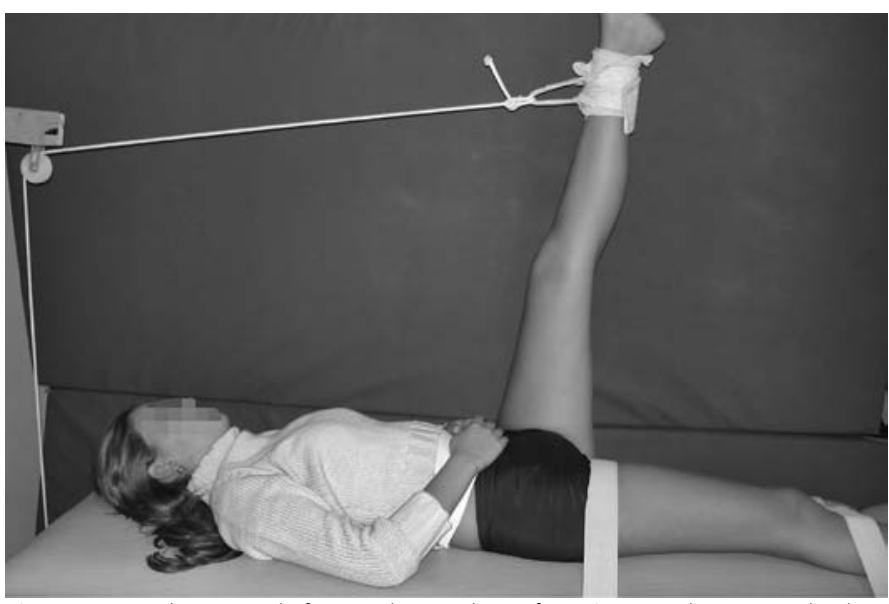

Figura 1. Foto ilustrativa da fixação do membro inferior à maca, da posição do alongamento e da polia com a corda presa ao tornozelo da voluntária. 


\section{RESULTADOS}

A massa corpórea das voluntárias com média e desvio padrão em cada grupo foi: GC (60,64 \pm 7,35); G30 (56,84 \pm 10,02); G3 (50,58 \pm $6,69)$. Os valores médios dos pesos e seus respectivos desvios padrão utilizados para o alongamento foram: G30: 5,71 \pm 1,03 e G3: 5,11 \pm 0,60 . Não houve diferença entre a carga utilizada para o alongamento nos dois grupos (G30 e G3) ( $p>0,05$ ).

A homogeneidade entre os grupos no início da pesquisa e os resultados relacionados com a amplitude de movimento do joelho nos grupos de estudo (G30 e G3) nas três avaliações (Av1, Av2 e Av3) encontram-se sumarizados na tabela 1.

Tabela 1. Valores médios \pm desvio padrão e valores $p$ da comparação das médias do ângulo das articulações do joelho dos grupos de estudo nas avaliações 1, 2 e 3.

\begin{tabular}{|c|c|c|c|}
\hline \multirow{2}{*}{ Grupos de estudo } & \multicolumn{3}{|c|}{ Avaliação } \\
\hline & Av1 & Av2 & Av3 \\
\hline Controle (GC) (N = 9) & $38,82^{\circ} \pm 6,08^{A ; a}$ & $36,67^{\circ} \pm 9,3^{\mathrm{Aia}}$ & $38,93^{\circ} \pm 9,79^{\mathrm{A} ; \mathrm{a}}$ \\
\hline $30 \mathrm{~s}(\mathrm{G} 30)(\mathrm{N}=7)$ & $45,05^{\circ} \pm 9,03^{\mathrm{A} ; \mathrm{a}}$ & $59,19^{\circ} \pm 7,13^{\mathrm{B} ; b}$ & $58,58^{\circ} \pm 11,23^{B ; b}$ \\
\hline $3 \min (\mathrm{G} 3)(\mathrm{N}=9)$ & $38,41^{\circ} \pm 8,90^{\mathrm{A} ; \mathrm{a}}$ & $50,34^{\circ} \pm 9,12^{\mathrm{B} ; \mathrm{b}}$ & $52,78^{\circ} \pm 7,98^{\mathrm{B} ; \mathrm{b}}$ \\
\hline \multicolumn{4}{|c|}{$\begin{array}{l}\text { Teste do efeito entre grupos: } F=7,397, g l=2, p=0,003 ; \\
\text { Teste do efeito intra-avaliações: } F=14,531, g l=2, p<0,001 ; \\
\text { Teste da interação: } F=4,794 ; g l=4, p=0,003 ; \\
\text { * Medidas seguidas com mesma letra minúsculas nas linhas não diferem significativamente entre os } \\
\text { tempos ( } p>0,05 \text { ). } \\
\text { * Medidas seguidas com a mesma letra maiúsculas nas colunas não diferem significativamente entre } \\
\text { os grupos ( } p>0,05 \text { ). }\end{array}$} \\
\hline
\end{tabular}

A figura 2 apresenta as alterações dos ângulos articulares (Av2-Av1) dos três grupos de estudo ilustrando o aumento de flexibilidade nos grupos $\mathrm{G} 30$ e $\mathrm{G} 3$.

A figura 3 apresenta as alterações dos ângulos articulares (Av3-Av1) dos três grupos de estudo ilustrando o aumento de flexibilidade nos grupos G30 e G3.

\section{DISCUSSÃO}

Mudanças no comprimento e extensibilidade muscular são as maiores causas das disfunções de movimento. Para o aumento da flexibilidade, o alongamento pode ser usado como um método de recuperação de $\mathrm{ADM}^{(3)}$. Pinfildi et al. ${ }^{(5)}$ relatam que a musculatura isquiotibial é uma das mais encurtadas devido ao sedentarismo. Além disso, Thacker et al.(1) e Davis et al. ${ }^{(2)}$ citam o alongamento estático como o mais efetivo para o aumento de flexibilidade desse grupo muscular, justificando a teoria de que o alongamento estático lento facilita o disparo do órgão tendinoso de Golgi (OTG), que produz a inibição do músculo alongado. A explicação neurofisiológica sugere que o fator limitante durante o alongamento é a resistência muscular secundária a uma atividade reflexa. O mecanismo para aumentar a amplitude de movimento é a mudança nas propriedades viscoelásticas do músculo(17). Se o alongamento ultrapassa o limite elástico, ponto além do qual o tecido não retorna ao seu formato e tamanho originais, ocorrerá deformação permanente ou plástica. Quando esse ponto é alcançado ocorre remodelamento do músculo(18).

Para a realização do trabalho, foram escolhidas apenas voluntárias do sexo feminino com o intuito de haver menos variabilidade quanto aos resultados observados. Achour Junior ${ }^{(6)}$ explica que a maior quantidade do hormônio estrógeno no sexo feminino causa menor desenvolvimento da massa muscular e maior concentração de água e polissacarídeos do que no sexo masculino, diminuindo o atrito entre as fibras musculares e, dessa forma, as condições de flexibilidade são maiores para as mulheres.

Para determinação da porcentagem de peso colocado para o alongamento em polias, alguns estudos foram tidos como base. Wessling et al. ${ }^{(16)}$ realizaram o alongamento de tríceps sural e utilizaram um peso fixo de 23,13 quilos para o alongamento com polias. Peres et al. ${ }^{(15)}$ também realizaram o alongamento da musculatura posterior da perna com o sistema de polias. Sugeriram que a utilização de um mesmo

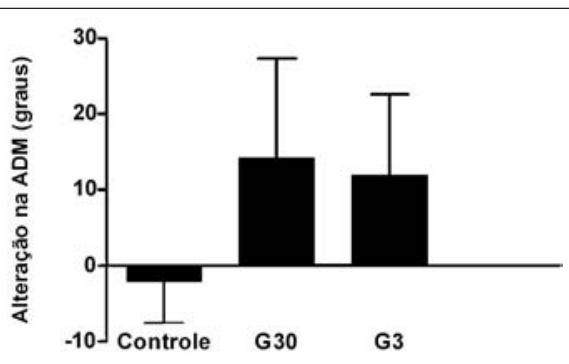

Figura 2. Valores médios \pm desvio padrão da alteração da amplitude de movimento nos três grupos de estudo ao término do protocolo (Av2).

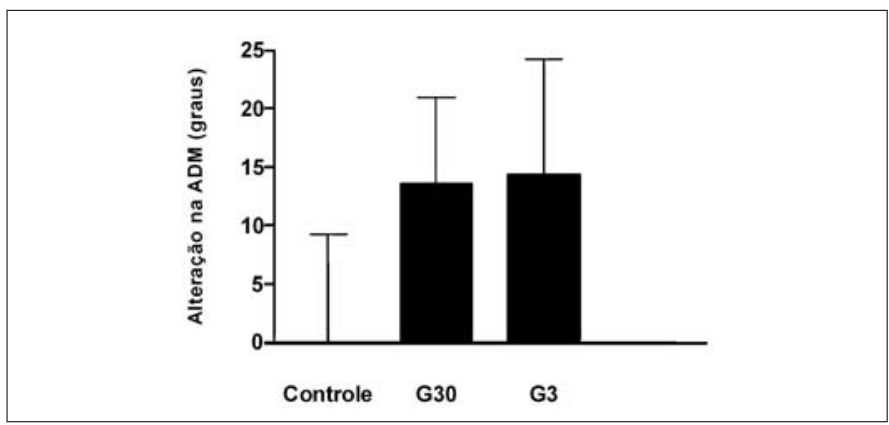

Figura 3. Valores médios \pm desvio padrão da alteração da amplitude de movimento nos três grupos de estudo cinco meses após o término do protocolo (Av3).

peso fixo para todos os sujeitos poderia ser desconfortável para alguns e inefetivo para outros. Por esse motivo, utilizaram um terço da massa corporal de cada voluntário para a realização do alongamento. Além disso, sugeriram a aplicação do mesmo método para alongamento da musculatura isquiotibial, onde grandes aumentos de ADM são possíveis. Pinfildi et al.(5) utilizaram para a realização do alongamento estático passivo dos isquiotibiais um peso fixo de sete quilos para todas as voluntárias. Sendo assim, o presente estudo realizou o alongamento dos isquiotibiais com o sistema de polias considerando 10\% da massa corporal de cada voluntária, sugerindo que esse peso pudesse causar tensão adequada para o alongamento dessa musculatura sem lesá-la, conforme os argumentos supracitados propostos por Peres et al. ${ }^{(15)}$.

A intervenção teve a duração de seis semanas, já que esse tempo se mostrou eficiente para o ganho da ADM causado pelo aumento da flexibilidade dos músculos isquiotibiais ${ }^{(3,7,8)}$. Foi escolhida a frequência de três vezes por semana, pois esta foi encontrada diversas vezes na literatura pesquisada e também se mostrou efetiva para aumento de flexibilidade $(2,7,9,19,20)$.

Precedido à intervenção, realizou-se o aquecimento na bicicleta ergométrica, sem carga, durante cinco minutos, com o intuito de prevenir lesões que poderiam ser causadas pelo alongamento ${ }^{(1,3,19,21)}$. Chan et al. ${ }^{(19)}$ realizaram, em seus grupos de intervenção de alongamento estático dos músculos isquiotibiais, aquecimento com duração de cinco minutos, igualmente ao presente estudo.

São diversos os estudos realizados a fim de caracterizar o tempo ideal de alongamento estático necessário para provocar mudança na ADM das articulações envolvidas, porém, são escassos os que utilizam o alongamento estático mantido por tempos maiores que um minuto. Bandy et al. ${ }^{(8)}$ utilizaram o alongamento estático mantido por séries de 30 segundos e um minuto e constataram aumento da flexibilidade dos músculos isquiotibiais, em relação ao pré e pós-teste de ambos os grupos, sem diferença estatisticamente significante entre eles. Os autores sugerem que o uso de longas durações e maior frequência de alongamento por dia deve ser questionado. Também sugerem que a manutenção do aumento da flexibilidade seja examinada. Chan et al. ${ }^{(19)}$ citam em seu estudo que maior tempo de alongamento mantido, provocando aumento da ADM, pode ser um indicativo de mudanças nas propriedades viscoelásticas e na manutenção da ADM adquirida. 
Tanto 10 séries de 30 segundos de alongamento quanto três séries de três minutos foram capazes de aumentar a ADM de joelho quando comparados com o controle. Thacker et al. ${ }^{(1)}$ sugerem que aumentos duráveis no comprimento muscular irão resultar de remodelamento adaptativo e não simplesmente da deformação viscoelástica. O presente estudo demonstrou que, após o término da intervenção proposta, o alongamento mantido por três minutos (três séries) e o alongamento mantido por 30 segundos (10 séries), em um programa de aumento de flexibilidade realizado três vezes por semana durante seis semanas, são capazes de provocar adaptação estrutural na musculatura posterior da coxa. A indagação que motivou a realização deste trabalho foi como otimizar 10 minutos de alongamento em uma sessão de fisioterapia ou durante a prática de uma atividade física ${ }^{(22)}$. Seria melhor usar maior numero de repetições com menor duração (10 X 30 segundos) ou usar menos repetições com maior duração ( 3 X 3 minutos)? Deve-se considerar que alongar por três vezes de três minutos seja mais prático que 10 vezes de 30 segundos, porém, podendo causar maior desconforto.

Não foi encontrada diferença entre os grupos G30 e G3 ao final do estudo. Morgan e Proske ${ }^{(23)}$ citam que mudanças no comprimento do músculo com o exercício em poucos dias são designadas por mudanças no número de sarcômeros em série. Relatam ainda que o ótimo comprimento muscular seja dado pelo comprimento dos tendões, pelo número de sarcômeros em série e pelo ótimo comprimento do sarcômero. Essa teoria pode explicar o motivo pelo qual não houve diferença entre os grupos, uma vez que tanto 10 séries de 30 segundos quanto três séries de três minutos de alongamento podem ser capazes de aumentar o número de sarcômeros em série. Três séries de alongamento mantido por 30 segundos parecem ser suficientes para provocar as mudanças no comprimento muscular, sem que seja necessário mantê-lo por mais tempo. Além disso, tempos adicionais não parecem trazer maiores benefícios na ativação dos componentes musculares envolvidos no aumento de flexibilidade.

Quanto à ADM de joelho adquirida (após cinco meses), os grupos G30 e G3 apresentaram resultados semelhantes quando comparados com o controle, evidenciando que o alongamento passivo estático mantido por 30 segundos (10 séries) ou por três minutos (três séries) obtém os mesmos resultados no que se refere à manutenção da flexibilidade adquirida na articulação do joelho, contrariando os achados de Lima et al. ${ }^{(3)}$. Em seu estudo, Lima et al. ${ }^{(3)}$, após a realização de um programa de flexibilidade, utilizando o alongamento estático dos músculos isquiotibiais durante seis semanas, executando quatro séries de 30 segundos, com e sem aquecimento prévio, relataram que, em aproximadamente 72 horas após o término da intervenção, os grupos de alongamento perderam os ganhos de ADM obtidos, sugerindo que não houve a adap- tação estrutural do músculo. 0 fato de esses autores terem utilizado menor número de séries de 30 segundos talvez explique a diferença dos resultados obtidos no presente estudo. Dessa forma, é de extrema importância o número de séries de alongamento realizadas e não apenas a duração de cada série. Poucas séries de 30 segundos parecem não gerar o volume de treino ideal para a manutenção da flexibilidade adquirida ${ }^{(3)}$. Em estudo parecido com o de Lima et al.(3), Weijer et al. ${ }^{(24)}$ realizaram também o alongamento por três séries de 30 segundos com e sem aquecimento prévio e constataram que ambos os grupos foram capazes de manter a amplitude de movimento adquirida após 24 horas. Se mais séries de alongamento de 30 segundos, tanto no estudo de Lima et al. ${ }^{(3)}$ quanto no de Weijer et al. ${ }^{(24)}$ tivessem sido realizadas, mensurações da flexibilidade após mais horas do término do protocolo poderiam ter indicado que a ADM se manteve como no presente estudo.

Não se sabe ao certo a explicação fisiológica que justifique o porquê de a amplitude de movimento ter se mantido por cinco meses, necessitando de outros tipos de estudo experimentais para que essa seja estabelecida. Considerando o princípio da sobrecarga do treinamento desportivo, a flexibilidade deveria ter sido reduzida. Porém, sabe-se que outros tecidos, além do muscular, influenciam o aumento da flexibilidade através do alongamento. Kisner e Colby ${ }^{(25)}$ citam que a cápsula articular é responsável por 47\%, o músculo por 41\%, o tendão por 10\% e a pele por $2 \%$. A deformação plástica dos tecidos conjuntivos deve ser considerada. A tensão passiva influencia a deformação do comprimento dos tecidos conjuntivos endomísio, perimísio e epimísio do ventre muscular, sendo o perimísio o maior contribuidor da resistência extracelular para o alongamento passivo(25). Sendo assim, a deformação plástica do colágeno pode justificar a durabilidade dos efeitos.

O estudo proposto apresentou algumas limitações, que devem ser consideradas em estudos futuros. Não foi controlada a intensidade do aquecimento pré-alongamento no cicloergômetro, através das rotações por minuto. A reprodutibilidade das medidas também não foi testada através do teste de confiabilidade intraexaminador, apesar de o avaliador ter sido cego e a média de três avaliações da ADM ter sido a utilizada.

\section{CONCLUSÃO}

Dez séries de 30 segundos e três séries de três minutos de alongamento podem aumentar a flexibilidade dos isquiotibiais e manter a flexibilidade adquirida após cinco meses do término da intervenção. Não foram observadas diferenças na flexibilidade adquirida com séries de 30 segundos e três minutos.

Todos os autores declararam não haver qualquer potencial conflito de interesses referente a este artigo.

\section{REFERÊNCIAS BIBLIOGRÁFICAS}

1. Thacker SB, Gilchrist J, Stroup DF, Kimsey CD Jr. The impact of stretching on sports injury risk: a systematic review of the literature. Med Sci Sports Exerc. 2003; 36:371-8.

2. Davis DS, Ashby PE, McCale KL, McQuain JA, Wine JM. The effectiveness of 3 stretching techniques on hamstring flexibility using consistent stretching parameters. J Strength Cond Res. 2005;19:27-32.

3. Lima RCM, Pessoa BF, Martins BLT, Freitas DBN. Análise da durabilidade do efeito do alongamento muscular dos isquiotibiais em duas formas de intervenção. Acta Fisiatr. 2006;13:33-9.

4. Spernoga SG, Uhl TL, Arnold BL, Gansneder BM. Duration of maintained hamstring flexibility after a one-time, modified hold-relax stretching protocol. J Athl Train. 2001;36:44-8.

5. Pinfildi CE, Prado RP, Liebano RE. Efeito do alongamento estático após diatermia de ondas curtas versus alongamento estático nos músculos isquiotibiais em mulheres sedentárias. Fisioter Bras. 2004;5:119-24.

6. Achour Junior A. Bases para exercícios de alongamento: relacionando com a saúde e no desempenho atlético. $2^{\mathrm{a}}$ ed. Londrina: Phorte, 1999.

7. Bandy WD, Irion JM. The effect of time on static stretch on the flexibility of the hamstring muscles. Phys Ther 1994;74:845-9.

8. Bandy WD, Irion JM, Briggler M. The effect of time and frequency on static stretching on flexibility of the hamstring muscles. Phys Ther. 1997;77:1090-6.

9. Roberts JM, Wilson K. Effect of stretching duration on active and passive range of motion in the lower extremity Br J Sports Med. 1999;33:259-63

10. Decoster LC, Cleland J, Altieri C, Russell P. The effects of hamstring stretching on range of motion: a systematic literature review. J Orthop Sports Phys Ther. 2005;35:377-87.

11. DePino GM, Webright WG, Arnold BL. Duration of maintained hamstring flexibility after cessation of an acute static stretching protocol. J Athl Train. 2000;35:56-9.

12. Magnusson SP, Aagard P, Simonsen E, Bojsen-Moller F. A biomechanical evaluation of cyclic and static stretch in human skeletal muscle. Int J Sports Med. 1998;19:310-6.

13. Borms J, Van Roy P, Santens JP, Haentjens A. Optimal duration of static stretching exercises for improvement of coxo-femoral flexibility. J Sports Sci. 1987;5:39-47.

14. Bonvicine C, Gonçalves C, Batigália F. Comparação do ganho de flexibilidade isquiotibial com diferentes técnicas de alongamento passivo. Acta Fisiatr. 2005;12:43-7.

15. Peres SE, Draper DO, Knight KL, Ricard MD. Pulsed shortwave diathermy and prolonged long-duration stretching increase dorsiflexion range of motion more than identical stretching without diathermy. J Athl Train. 2002;37:43-50.

16. Wessling KC, DeVane DA, Hylton CR. Effects of static stretch versus static stretch and ultrasound combined on triceps surae muscle extensibility in healthy women. Phys Ther. 1987;67:674-9.

17. Magnusson SP. Passive properties of human skeletal muscle during stretch maneuvers. Scand J Med Sci Sports. 1998;8:65-77.

18. Kubo K, Kanehisa H, Fukunaga T. Effect of stretching training on the viscoelastic properties of human tendon structures in vivo. J Appl Physiol. 2002;92:595-601.

19. Chan SP, Hong Y, Robinson PD. Flexibility and passive resistance of the hamstrings of young adults using two different static stretching protocols. Scand J Med Sci Sports. 2001;11:81-6.

20. Draper DO, Miner L, Knight KL, Ricard MD. The carry-over effects of diathermy and stretching in developing hamstring flexibility. J Athl Train. 2002;37:37-42.

21. Handel M, Horstmann T, Dickhurt HH, Gulch RW. Effects of contract-relax stretching training on muscle performance in athletes. Eur J Appl Physiol Occup Physiol. 1997;76:400-8.

22. Halbertsma JPK, Goeken LNH. Stretching exercises: effect on passive extensibility and stiffness in short hamstrings of healthy subjects. Arch Phys Med Rehabil. 1994;75:976-81.

23. Morgan DL, Proske U. Popping sarcomere hypothesis explains stretch-induced muscle damage. Clin Exp Pharmacol Physiol. 2004;31:541-7.

24. Weijer VC, Gorniak GC, Shamus E. The effect of static stretch and warm-up exercise on hamstring length over the course of 24 hours. J Orthop Sports Phys Ther. 2003;33:727-33.

25. Kisner C, Colby LA. Exercícios terapêuticos: fundamentos e técnicas. 3a ed. São Paulo: Manole, 1998. 